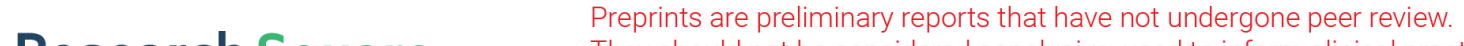 They should not be considered conclusive, used to inform clinical practice, or referenced by the media as validated information. \\ The Therapeutic and Prognostic Value of Chemokine Receptors (CXCRs) in Skin Cutaneous Melanoma (SKCM) Progression
}

\section{Sumei Huang}

Maoming People's Hospital

\section{Guangwen Wang}

Maoming People's Hospital

\section{Mengying Kou}

Maoming People's Hospital

Yisheng Huang

Maoming People's Hospital

He Yanhong

Maoming People's Hospital

Bai Yunpeng

Maoming People's Hospital

\section{Li Yingbang}

Maoming People's Hospital

Wendong Huang ( $\square$ huangwendong418@126.com )

Maoming People's Hospital https://orcid.org/0000-0002-6119-5847

\section{Research Article}

Keywords: Skin cutaneous melanoma (SKCM), CXC chemokine receptors (CXCRs), biomarker, bioinformatics analysis, tumor microenvironment

Posted Date: June 22nd, 2021

DOI: https://doi.org/10.21203/rs.3.rs-618975/v1

License: (c) (1) This work is licensed under a Creative Commons Attribution 4.0 International License. Read Full License 


\section{Abstract}

Introduction:Skin cutaneous melanoma (SKCM) is an aggressive cancer and associates with CXC chemokine receptors (CXCRs). CXCRs is a group of membrane protein involved in tumor initiation, progression, and outcome. However, the therapeutic and prognostic value of CXCRs in SKCM remained elusive.

Methods: we used multiple, publicly available datasets for the analysis of CXCRs in SKCM. These datasets included GEPIA, UALCAN, TISIDB, DAVID 6.8, Metascape, GSCALite, and TIMER.

Results: $C X C R 3$ and $C X C R 4$ were elevated in tumor samples in comparison to healthy ones. $C X C R 3$, CXCR4, CXCR5, CXCR6, and CXCR7 were transcriptionally upregulated in the metastasis SKCM tissues compared to the primary tissues. The $C X C R 3, C X C R 4, C X C R 5$, and $C X C R 6$ were positively correlated to more prolonged overall survival (OS) at the transcriptional level. Meanwhile, CXCR3, CXCR4, and CXCR6 had an association with the stage in SKCM patients. The drug sensitivity analysis showed CXCR4 as a potential target of drug screening. The function of CXCRs and the neighboring genes mainly enriched in T cell activation, cytokine-cytokine receptor interaction, and leukocyte migration. Cancer pathway analysis showed that all the CXCRs positively affect apoptosis and EMT pathway and negative effect on the cell cycle, DNA damage response, and hormone AR pathway. Furthermore, CXCR3, CXCR4, CXCR5, and CXCR6 were positively correlated with the infiltration of lymphocytes and expression of PD-1, PD-L1, and CTLA-4.

Conclusions: we found that $C X C R 3, C X C R 4, C X C R 5$, and CXCR6 may provide essential clues about credible prognostic biomarkers, especially $C X C R 4$ may be a potential target and prognostic biomarkers in the progression of SKCM.

\section{Introduction}

Skin cutaneous melanoma (SKCM) is a malignant tumor disease resulting in most of the mortality rate among skin tumors (1). This disease's incidence is 15-25 per 100,000 individuals, which is increasing faster than any other cancers (2). The metastatic SKCM has a $15-20 \%$ five-year survival rate (3). So the most pressing need is to excavate and identify biomarkers to predict the prognosis and treat the patients diagnosed with SKCM (4).

SKCM is a high immunogenicity tumor infiltrated with various immune cells (5). Chemokines and their receptors help to relocate the immune cells into the areas of cancer cells to eliminate tumor cells, which may affect the process of SKCM (6). Specific expression of chemokine receptors led to changes in the stage of cancer homing and metastasis dependent on receptor and organ (7). There are four classes of chemokine receptors, CXCR, CCR, CR, and CX3CR, named with their corresponding Chemokines (8). Most recently, CXCRs exert essential functions in melanoma progression (9). There are seven subtypes of CXCRs, respectively were named CXCR1, CXCR2, CXCR3, CXCR4, CXCR5, CXCR6, CXCR7 (also known as ACKR3). It was reported that $C X C R 1$ or $C X C R 2$ were stably overexpressed in human melanoma cell and 
enhanced the proliferation, motility and invasion of tumor cells (10). CXCR3 was upregulated in human vertical growth phase melanoma, closely correlating with increased metastasis and poor OS (11). Murakami et al. reported that CXCR4 could enhance pulmonary metastases, which indicated that chemokine receptors were involved in the mechanism of tumor metastasis (12). A growing body of evidence showed that $C X C R 7$ expression was elevated in a variety of malignancies and shows multiple functions in tumor progression (13). In murine melanoma cell lines $C X C R 7$ was overexpressed and high expression of $C X C R 7$ was associated with bad overall survival in melanoma patients. Many studies described the functions and prognostic impact of individual CXCRs in melanoma. However, there are few systematic investigations of the therapeutic and prognostic value of CXCRs in SKCM.

With the comprehensive development of second-generation sequencing technology, the establishment of various databases and the development of visual websites have made it possible to comprehensively analyze mRNA levels in various cancers. Here we performed a comprehensive study on CXCRs based on several large public databases to investigate how much CXCRs contribute to prognosis. The aim was to provide essential clues about credible prognostic biomarkers and explore effective treatment to prevent cancer deterioration.

\section{Methods}

\subsection{GEPIA}

GEPIA (http://gepia.cancer-pku.cn/index.html) is an effective tool for revealing the meaningful information of the large-scale genomic data in TCGA and GTEx, which contained RNA sequence expression data of 9,736 tumors and 8,587 standard tissue samples (14). In this study, we performed an analyses of mRNA expression in tumors and healthy control tissues. Furthermore, we analyzed the mRNA expression of CXCRs in the pathological stages and its correlation to prognosis using the "Single Gene Analysis" module of GEPIA. Besides, we performed multiple gene comparison analysis of CXCRs with the "Multiple Gene Comparison" module of GEPIA by using the "KIRC" dataset. We used the student's t-test to determine whether expression and pathological stages were significantly different $(p<0.05)$. We used a Kaplan-Meier curve to analyze the prognosis.

\subsection{UALCAN}

UALCAN (http://ualcan.path.uab.edu/analysis.html) is an efficient platform to generate information on the expression of genes based on TCGA and MET500 cohort data (15). We obtained the transcriptional of CXCRs in primary and metastasis SKCM by using the "Expression Analysis" module of UALCAN $(p<0.05)$.

\subsection{TISIDB}

TISIDB (http://cis.hku.hk/TISIDB/) (16)is a web portal for tumor and immune system interaction, which integrates multiple heterogeneous data types. TISIDB was applied to investigate the correlation between the mRNA levels of CXCRs and different immune subtypes of SKCM by the Kruskal-Wallis test $(p<0.05)$. 


\subsection{DAVID 6.8}

DAVID 6.8 (https://david.ncifcrf.gov/home.jsp) is a data analysis website that provides the biological function of target genes (17). In our research, we analyzed the functions of differentially expressed CXCRs with DAVID 6.8. We got the gene enrichment information with the Gene Ontology (GO) and Kyoto Encyclopedia of Genes and Genomes (KEGG) pathway. GO analysis is a primary bioinformatics tool, can provide gene enrichment information, including Biological processes (BP), cellular components (CC), and molecular function (MF). Moreover, the visualization of this information was obtained with the R project ( $p$-value<0.05).

\subsection{Metascape}

Metascape (http://metascape.org) is a portal that provides a gene annotation and analysis of gene list enrichment (5). Metascape can promote data-driven decisions based on the functional list of genes/proteins. We undertook the Metascape analysis to elucidate the CXCRs further and their neighbor genes. "Custom Analysis" module was used to verify the functional annotation of CXCRs and their neighbor genes with min overlap of $3, p$-value $<0.01$ and min enrichment of 1.5.

\subsection{GSCLite}

GSCALite (http://bioinfo.life.hust.edu.cn/web/GSCALite/) is a platform for gene sets analysis (18). We used TCGA SKCM dataset to generate information on genetic alterations, cancer pathway activity, and drug sensitivity analysis of CXCRs.

\subsection{TIMER}

TIMER (https://cistrome.shinyapps.io/timer/) is a web server that can predict the infiltration of immune cells of cancers and their clinical impact (19). We analyzed CXCRs expression as a "Correlation module" to estimate the relationship between their level and the immune cell infiltration and immune checkpoints PD-1, PD-L1, and ctla-4 ( $p$-value $<0.05)$.

\section{Results}

\subsection{Aberrant Expression of CXCRs in Patients with SKCM.}

We detected the transcriptional levels of CXCRs in SKCM and healthy tissues with GEPIA (Fig. 1). The transcriptional levels of $C X C R 3$ and $C X C R 4$ in SKCM tissues were upregulated, while CXCR2, CXCR7, declined. Then, the transcriptional of CXCRs in primary SKCM and metastasis SKCM were evaluated with UALCAN (Fig. 2). The transcriptional levels of CXCR4, CXCR5, CXCR6, and CXCR7 were higher in the metastasis SKCM tissues than in the primary SKCM, while the transcriptional levels of CXCR1, CXCR2 and $C X C R 3$ in the metastasis SKCM tissues were lower than that in controls.

Subsequently, the TISIDB was used to investigate the correlation between the mRNA levels of CXCRs and different immune subtypes of SKCM (Fig. 3). We found that the expression of the CXCR3, CXCR4, CXCR5, 
and $C X C R 6$ was significant differences in the immune subtypes of SKCM, while the expression of the CXCR1, CXCR2, and CXCR7 showed no statistical difference. Interestingly the levels of CXCR3, CXCR4, CXCR5, and CXCR6 were improved in the IFN-gamma dominant of the SKCM immune subtype, while decreased in the lymphocyte depleted immune subtype.

\subsection{CXCRs correlate with the stage of SKCM and predict the Prognosis in Patients Diagnosed with SKCM}

We evaluated whether the expression of CXCRs was associated with the stage of SKCM using GEPIA (Fig. 4, A). The results showed that $C X C R 3(p=1.58 \mathrm{E}-03), C X C R 4(p=4.94 \mathrm{E}-06)$, and $C X C R 6(p=3.21 \mathrm{E}-$ 04 ) were significantly correlated with the cancer stage. In general, the expression of $C X C R 3, C X C R 4$, and CXCR6 in stage I was significantly higher than that in stage 0 , and their expression in stage II was markedly decreased, followed by an upward trend in stage $\nabla$ and $\otimes$, while no correlation was found between the level of CXCR1, CXCR2, CXCR5, and CXCR7 and the pathological stage of SKCM patients. Consistently, the expression levels of $C X C R 3, C X C R 4$, and CXCR6 were relatively low in Stage II compared with other stages. These data suggested that $C X C R 3, C X C R 4$, and CXCR6 were involved in the deterioration of SKCM,especially, they may be involved in important processes in Phase II.

Furthermore, we evaluated the differentially expressed CXCRs in the survival of SKCM patients to assess the functions of CXCRs in the progression of SKCM with GEPIA (Fig. 4, B). The results showed that the expression of $C X C R 1$ in SKCM patients was negatively correlated with survival time ( $p=3.7 \mathrm{E}-03)$. In contrast, $C X C R 3(p=1.6 \mathrm{E}-04), C X C R 4(p=2.1 \mathrm{E}-02), C X C R 5(p=0.01)$ and $C X C R 6(p=1.6 \mathrm{E}-05)$ in the SKCM patients were positively correlated to survival time. In other words, the patients with high expression of CXCR3, CXCR4, CXCR5, and CXCR6 might have longer overall survival.

\subsection{Functional Enrichment Analysis of CXCRs in SKCM}

To understand the function of distinct CXCRs in the development of SKCM, we isolated the top 71 CXCRs neighboring genes by using GEPIA to explore further the biological functions of CXCRs (Table 1). We conducted Enrichment analysis, including GO function and KEGG pathways, using DAVID 6.8 to analyze CXCRs and their neighboring genes (Fig. 5).

CXCRs and their neighboring genes were most enriched in T cell activation and leukocyte migration in the biological process (BP), receptor binding and activity in the molecular function (MF), external side of plasma membrane in the cellular component (CC), cytokine-cytokine receptor interaction in KEGG pathway analysis (Fig. 5, A-D). Moreover, the functions of CXCRs and their neighboring genes were further verified using the Metascape (Fig. 5, E). Broadly consistent with the DAVID 6.8 results, the most enriched functions were T cell activation, cytokine-cytokine receptor interaction, and leukocyte migration.

\subsection{Analyses of Genetic Alteration, Cancer Pathway Activity and Drug Sensitivity of CXCRs in SKCM patients}

We performed a comprehensive analysis of genetic alterations, pathway activity, and drug sensitivity analysis of CXCRs using the GSCALite. Around 50\% of SKCM samples showed genetic alterations, and 
the main mutations form was missense mutation. CXCR2 and CXCR 1 were altered in $42 \%$ and $31 \%$ of the queried SKCM samples, respectively (Fig. 6, A).

We explored the cancer pathway activity among the CXCRs by using GSCALite (Fig. 6, B). All the CXCRs positively affect the apoptosis and EMT (Epithelial-Mesenchymal Transition) pathway, and negatively affect the cell cycle, DNA damage response, and hormone AR pathway.

We carried out the drug sensitivity analysis with 265 small molecules from Genomics of Drug Sensitivity in Cancer (GDSC) by using GSCALite (Fig. 7). Low expression of CXCR4 showed resistance to most of the small molecules (blue point), while high expression of $C X C R 4$ was resistant to many anticancer drugs, including trametinib, telatinib (17-AAG), and docetaxel (orange point), which suggested CXCR4 might be a potential biomarker for drug screening.

\subsection{CXCRs involved in Immune Cell Infiltration in Patients with SKCM and were associated with immune checkpoints}

The anti-tumor activity was significantly correlated with the infiltration of immune factors in the tumor environment. We used TIMER to analyze CXCRs expression correlated with immune cells in SKCM. The expression of CXCRs members negatively related to tumor purity in SKCM (Fig. 8). Generally, CXCR3, CXCR4, CXCR5, and CXCR6 were positively correlated with the infiltration of B cells, CD $8+\mathrm{T}$ cells, CD $4+\mathrm{T}$ cells, macrophages, neutrophils, and dendritic cells. However, the expression of CXCR1 and CXCR2 was almost not correlated with the infiltration of immune factors, while the expression of $C X C R 7$ was only positively associated with the infiltration of CD $4+T$ cells, macrophages, and neutrophils.

The anti-tumor effect also depends on the expression of the immune checkpoints in tumor cells, so we also analyzed the relation of CXCRs expression and immune checkpoints (PD-1, PD-L1, and CTLA-4) in SKCM with TIMER (Fig. 9). Increased CXCR3, CXCR4, CXCR5, and CXCR6 were positively correlated with high expression of PD-1, PD-L1, and CTLA-4. Especially, the expression of CXCR3 and CXCR6 were strongly correlated with PD-1 expression ( $p=4.87 \mathrm{E}-43$ and $p=1.33 \mathrm{E}-27$ respectively), which may be a potential predictive biomarker for the sensitivity of Immunosuppressive therapy. Except for $C X C R 1$ and CXCR2 showed a weak positive correlation of PD1, there was no significant correlation between the expression of $C X C R 1, C X C R 2$, and $C X C R 7$ and the immune checkpoints expression levels.

\section{Discussion}

CXCRs, as one of the important subtypes of chemokine receptor, are involved in a variety of physiological functions such as cell growth, development, differentiation, apoptosis and distribution. Tumor microenvironment greatly affect the growth, local invasion, and organ-specific metastasis of tumor cells, and ultimately affects the outcome of tumor patients (20). Numerous studies have shown that CXCRs and their ligands affect the formation and metastasis of various tumors by change the infiltrating tumor microenvironment (21). However, the expression and the prognostic value of CXCRs in SKCM has not been systematically investigated. Here, we used large databases for the analysis of CXCRs in SKCM. 
Firstly, we found that CXCR3 and CXCR4 were highly expressed in SKCM compared to healthy tissue. These data were consistent with Jenkins et al (22). and Doron et al. (23). Meanwhile, mounting evidence manifested that $C X C R 4$ was overexpressed in melanomas throughout progression(24). The transcriptional level of $C X C R 7$ was a distinct decreased in SKCM tissues in our study. This result was inconsistent with Xu et al. (9), who found that $C X C R 7$ was overexpressed in murine melanoma cell lines. The opposite result may be due to differences in the species of the two studied. Chemokine receptor expression, including $C X C R 3$ and $C X C R 4$, is supposed to lead and enhance site-directed metastases (25). In our study, CXCR3, CXCR4, CXCR5, CXCR6, and CXCR7 were highly expressed in metastasis SKCM compared with primary SKCM. This result was consistent with the previous study, which reported the expression of CXCR5 and CXCR6 were significantly increased in metastases melanoma to the kidney and the cerebrum(24). Several reports showed that $C X C R 4$ could enhance tumor metastasis, though the mechanism still have no consensus(26). Thus, CXCR4 may be an effective therapeutic target for inhibiting tumor metastasis.

Credible prognostic biomarkers for primary melanomas are urgently needed to develop effective therapeutic strategies to prevent tumor migration and metastasis. In our study, the expression of $C X C R 3$, CXCR4, and CXCR6 in the State $\otimes$ to $\otimes$ of SKCM was significantly different from that of stage 0 , especially in stage II, these receptors displayed the lowest expression level, suggesting that $C X C R 3, C X C R 4$, and CXCR6 plays an important role in the progression of stage II disease. McConnell et al. found that CXCR4 expression of melanoma was significantly higher in stage $\nabla$ and $\nabla$ than that in stage $\nabla$ and $\nabla(25)$, which was not in consistent with our result.

Moreover, we investigated the prognostic value of CXCRs in patients with SKCM, as expected, the expression of CXCRs were associated with survival. CXCR3, CXCR4, CXCR5, and CXCR6 were positively associated with more prolonged overall survival. These results were in line with previous studies, which found that the high expression of $C X C R 3, C X C R 5$, and $C X C R 6$ were associated with better prognosis in melanoma and breast cancer(27). However, another report demonstrated Knockdown of chemokine receptor CXCR4 gene could inhibit the growth of B16F10 melanoma(28). McConnell et al. also found that primary cutaneous melanomas with high CXCR4 expression (> 50\%) were three-fold more likely to develop metastasis with concurrent reduced disease-free survival(29). The conflict in the OS rates among the present study and previous studies required larger sample-sizes and high-quality trials to show significance.

Genetic alteration plays an essential role in tumorigenesis and the progression of SKCM(30). In our study, alterations with the provisional datasets of TCGA showed the most alteration is elevated missense mutation. The cancer pathway activity analysis showed that CXCRs had an active effect on the apoptosis and EMT (Epithelial-Mesenchymal Transition) pathway and negative effect on the cell cycle, DNA damage response, and hormone AR (Androgen Receptor) pathway. The process of apoptosis, also called programmed cell death,was genetically regulated autonomous cell death. The abnormal apoptotic process causes the imbalance between cell division and death, leading to malignant transformation and the occurrence of tumor(31). EMT was the biological process of epithelial cells transforming into cells 
with a mesenchymal phenotype through a specific program. It played an important role in tumor progression and metastasis (32). Previous studies had found that CXCR7 controlled cell adhesion, migration, and proliferation by EMT pathway (33). Therefore, CXCRs might affect the occurrence, development, and metastasis of SKCM through these signaling pathways. Drug sensitivity analysis documented CXCR4 as a potential biomarker for drug screening, which showed resistance to 71 small molecules. Mountains of reports had indicated that CXCR4 was one of the most potential chemokine receptors in malignancy. Small moleculars (34) or antibodies (35) that inhibiting the activity of CXCR4 could reduce the metastasis of tumor cells in vitro and in vivo.

We investigated the function of differentially expressed CXCRs using GO enrichment analysis, KEGG pathway enrichment analysis, and Metascape analysis. The results showed that the most highly enriched functions were T cell activation, cytokine-cytokine receptor interaction, hematopoietic cell lineage, and leukocyte migration. Interaction between chemokines and receptors plays a vital role in immune cell differentiation, development, and directional migration (36). The CXCL12-CXCR4 axis is a crucial signal that regulates the hematopoietic stem cell compartment in the bone marrow (37). Several studies show CXCRs signaling pathways play an essential role in immune activation (38), senescence (39), immune evasion (40), and metastasis of various cancers $(41,42)$.

In many malignancies, enhanced infiltration of the tumor by an immune cell is typically associated with a good prognosis (6). Intense infiltration, especially by cytotoxic CD $8+T$ cells, is usually associated with favorable OS (43). Our result showed that CXCR3, CXCR4, CXCR5, and CXCR6 had a high correlation with CD8 + T cells, CD $4+T$ cells, neutrophils, and dendritic cells. Meanwhile, the expression of CXCR1, CXCR2 was almost not correlated with the infiltration of immune factors, while the expression of $C X C R 7$ was only positively associated with the infiltration of CD4 + T cells, macrophages, and neutrophils. These results combined with the survival of SKCM suggested that the infiltration of immune cells might be associated with better survival. Chen et al. also had similar results (44), who found that the high expression of $C X C R 3$ in gastric cancer (GC) was positively correlated with M2 macrophage infiltration and improved survival. Meanwhile, Saxena et al. found that CXCR4 antagonist exert anti-tumor effects by increasing the infiltration of CD8 + cells and improve the survival rate of patients (45).

CTLA-4, PD-1, and PD-L1 immune checkpoint inhibitors have been shown to prolong survival in metastatic melanoma (45). Similar trends were observed with the infiltration of immune cell, overexpression of $C X C R 3, C X C R 4, C X C R 5$, and $C X C R 6$ were positively correlated with high expression of PD-1, PD-L1, and CTLA-4, while no significant correlation was observed between the expression of $C X C R 1$, CXCR2, and $C X C R 7$ and the immune checkpoints expression levels, which suggested that $C X C R 3, C X C R 4$, CXCR5, and CXCR6 might enhance the antitumor effect of immune checkpoint inhibitors (ICls). Saxena et al. (45)found that $C X C R 4$ antagonist X4-136 combination with checkpoint inhibitors axitinib inhibited the growth of melanoma and renca tumors, thought increasing the infiltration of CD8+ / perforin + cells. It was suggested that CXCRs or their blockers combined with immune checkpoint inhibitors might be a novel approach strategy for the treatment of cancer. 
There are some limitations to our study. First, GPR35, the receptor of CXCL17 was recently renamed as CXCR8 (46). However due to a lack of data in the database, this receptor was not included in this study. Then, the analysis on the transcriptional level cannot reflect global changes in the immune status. Furthermore, more in vitro or in vivo studies should be performed to validate our results.

\section{Conclusion}

We define the contribution of CXCRs in the SKCM. We found that CXCR3, CXCR4, CXCR5, and CXCR6 may provide a diagnostic and prognostic role in SKCM. Besides, $C X C R 4$ could serve as a promising target of drug design and treatment.

\section{Abbreviations}

SKCM

Skin cutaneous melanoma; CXCRs:CXC chemokine receptors; OS:Overall survival; GO:Gene Ontology; KEGG:Kyoto Encyclopedia of Genes and Genomes; BP:Biological processes; CC:Cellular components; MF:Molecular function; PPI:Protein-protein interaction; EMT:Epithelial-Mesenchymal Transition; Hormone AR:Androgen Receptor; GDSC:Genomics of Drug Sensitivity in Cancer; GC:gastric cancer; ICls $\square$ immune checkpoint inhibitors.

\section{Declarations}

\section{Acknowledgments}

This work was supported by the High-level Hospital Construction Research Project of Maoming People's Hospital; Medical Science and Technology Research Foundation of Guangdong Province (Project No. B2020194); China Postdoctoral Foundation (Project No. 2018m633286); Science and Technology Plan Project of Maoming (Project No. 2019018023), Medical Science and Technology Research Foundation of Guangdong Province (Project No. B2018061 and A2017490).

\section{Authors' contributions}

Sumei Huang designed, revised, and finalized the manuscript. Sumei Huang, Guangwen Wang and Mengying Kou were the first authors who contributed to the drafting and editing of the manuscript. Guangwen Wang prepared the figures and tables. Mengying Kou contributed to literature search. Yanhong, He and Yingbang, Li participated in the drafting and editing of the manuscript. Yisheng Huang, Yunpeng, Bai, and Wendong Huang participated in the revision and coordination. All authors read and approved the final manuscript.

\section{Funding}


High-level Hospital Construction Research Project of Maoming People's Hospital; Medical Science and Technology Research Foundation of Guangdong Province (Project No. B2020194); China Postdoctoral Foundation (Project No. 2018m633286); Science and Technology Plan Project of Maoming (Project No. 2019018023); Medical Science and Technology Research Foundation of Guangdong Province (Project No. B2018061 and A2017490).

\section{Availability of data and materials}

All data generated or analyzed during this study are included in this published article.

\section{Ethics approval and consent to participate}

This was not applicable to this manuscript.

\section{Consent for publication}

Consent for publication was obtained from all participants.

\section{Competing interests}

The authors declare that they have no competing interests.

\section{References}

1. Qin $Y$, Verdegaal E, Siderius M, Bebelman J, Smit M, Leurs R, et al. Quantitative expression profiling of G-protein-coupled receptors (GPCRs) in metastatic melanoma: the constitutively active orphan GPCR GPR18 as novel drug target. Pigment cell melanoma research. 2011;24(1):207-18.

2. Thompson J, Scolyer R, Kefford R. Cutaneous melanoma. Lancet. 2005;365(9460):687-701.

3. van Rooijen E, Fazio M, Zon L. From fish bowl to bedside: The power of zebrafish to unravel melanoma pathogenesis and discover new therapeutics. Pigment cell melanoma research. 2017;30(4):402-12.

4. Zhang W, Zhao H, Chen J, Zhong X, Zeng W, Li Z, et al. Mining database for the expression and gene regulation network of JAK2 in skin cutaneous melanoma. Life sciences. 2020;253:117600.

5. Zhao Y, Schaafsma E, Gorlov I, Hernando E, Thomas N, Shen R, et al. A Leukocyte Infiltration Score Defined by a Gene Signature Predicts Melanoma Patient Prognosis. Molecular cancer research: MCR. 2019;17(1):109-19.

6. Xiong T, Pan F, Liang Q, Luo R, Li D, Mo H, et al. Prognostic value of the expression of chemokines and their receptors in regional lymph nodes of melanoma patients. $\mathrm{J}$ Cell Mol Med. 2020;24(6):3407-18.

7. Balkwill F. Cancer and the chemokine network. Nature reviews Cancer. 2004;4(7):540-50.

8. Robledo M, Bartolome R, Longo N, Rodríguez-Frade J, Mellado M, Longo I, et al. Expression of functional chemokine receptors CXCR3 and CXCR4 on human melanoma cells. J Biol Chem. 
2001;276(48):45098-105.

9. Xu S, Tang J, Wang C, Liu J, Fu Y, Luo Y. CXCR7 promotes melanoma tumorigenesis via Src kinase signaling. Cell death disease. 2019;10(3):191.

10. Singh S, Nannuru K, Sadanandam A, Varney M, Singh R. CXCR1 and CXCR2 enhances human melanoma tumourigenesis, growth and invasion. British journal of cancer. 2009;100(10):1638-46.

11. Kawada K, Sonoshita M, Sakashita H, Takabayashi A, Yamaoka Y, Manabe T, et al. Pivotal role of CXCR3 in melanoma cell metastasis to lymph nodes. Cancer research. 2004;64(11):4010-7.

12. Murakami T, Maki W, Cardones A, Fang H, Tun Kyi A, Nestle F, et al. Expression of CXC chemokine receptor-4 enhances the pulmonary metastatic potential of murine B16 melanoma cells. Cancer research. 2002;62(24):7328-34.

13. Miao Z, Luker K, Summers B, Berahovich R, Bhojani M, Rehemtulla A, et al. CXCR7 (RDC1) promotes breast and lung tumor growth in vivo and is expressed on tumor-associated vasculature. Proc Natl Acad Sci USA. 2007;104(40):15735-40.

14. Tang Z, Li C, Kang B, Gao G, Li C, Zhang Z. GEPIA: a web server for cancer and normal gene expression profiling and interactive analyses. Nucleic acids research. 2017;45:W98-102.

15. Wu G, Zhang Z, Tang Q, Liu L, Liu W, Li Q, et al. Study of FABP's interactome and detecting new molecular targets in clear cell renal cell carcinoma. Journal of cellular physiology. 2020;235(4):3776-89.

16. Ru B, Wong C, Tong Y, Zhong J, Zhong S, Wu W, et al. TISIDB: an integrated repository portal for tumor-immune system interactions. Bioinformatics. 2019;35(20):4200-2.

17. Huang dW, Sherman B, Lempicki R. Systematic and integrative analysis of large gene lists using DAVID bioinformatics resources. Nature protocols. 2009;4(1):44-57.

18. Yuemaier M, Zhou Z, Zhou Y, Wu C, Li F, Liang X, et al. Identification of the Prognostic Value and Clinical Significance of Interferon Regulatory Factors (IRFs) in Colon Adenocarcinoma. Medical science monitor: international medical journal of experimental clinical research. 2020;26:e927073.

19. Li T, Fan J, Wang B, Traugh N, Chen Q, Liu J, et al. TIMER: A Web Server for Comprehensive Analysis of Tumor-Infiltrating Immune Cells. Cancer research. 2017;77(21):e108-e10.

20. Zhu Q, Han X, Peng J, Qin H, Wang Y. The role of CXC chemokines and their receptors in the progression and treatment of tumors. J Mol Histol. 2012;43(6):699-713.

21. Richmond A, Yang J, Su Y. The good and the bad of chemokines/chemokine receptors in melanoma. Pigment cell melanoma research. 2009;22(2):175-86.

22. Jenkins $M$, Brinckerhoff $C$, Mullins D. CXCR3 signaling in BRAFWT melanoma increases IL-8 expression and tumorigenicity. PloS one. 2015;10(3):e0121140.

23. Doron H, Amer M, Ershaid N, Blazquez R, Shani O, Lahav T, et al. Inflammatory Activation of Astrocytes Facilitates Melanoma Brain Tropism via the CXCL10-CXCR3 Signaling Axis. Cell reports. 2019;28(7):1785-98.e6. 
24. Seidl H, Richtig E, Tilz H, Stefan M, Schmidbauer U, Asslaber M, et al. Profiles of chemokine receptors in melanocytic lesions: de novo expression of CXCR6 in melanoma. Human pathology. 2007;38(5):768-80.

25. McConnell A, Ellis R, Pathy B, Plummer R, Lovat P, O'Boyle G. The prognostic significance and impact of the CXCR4-CXCR7-CXCL12 axis in primary cutaneous melanoma. Br J Dermatol. 2016;175(6):1210-20.

26. Müller A, Homey B, Soto H, Ge N, Catron D, Buchanan M, et al. Involvement of chemokine receptors in breast cancer metastasis. Nature. 2001;410(6824):50-6.

27. Lyu $L$, Zheng $Y$, Hong $Y$, Wang $M$, Deng $Y, W u Y$, et al. Comprehensive analysis of the prognostic value and immune function of chemokine-CXC receptor family members in breast cancer. Int Immunopharmacol. 2020;87:106797.

28. André N, Silva V, Watanabe M, De Lucca F. Knockdown of chemokine receptor CXCR4 gene by RNA interference: Effects on the B16-F10 melanoma growth. Oncol Rep. 2016;35(4):2419-24.

29. McConnell A, Ellis R, Pathy B, Plummer R, Lovat P, O'Boyle G. The prognostic significance and impact of the CXCR4-CXCR7-CXCL12 axis in primary cutaneous melanoma. Br J Dermatol. 2016;175(6):1210-20.

30. Zhao X, Little P, Hoyle A, Pegna G, Hayward M, Ivanova A, et al. The Prognostic Significance of LowFrequency Somatic Mutations in Metastatic Cutaneous Melanoma. Frontiers in oncology. 2018;8:584.

31. Goldar S, Khaniani M, Derakhshan S, Baradaran B. Molecular mechanisms of apoptosis and roles in cancer development and treatment. Asian Pacific journal of cancer prevention: APJCP. 2015;16(6):2129-44.

32. Hodorogea A, Calinescu A, Antohe M, Balaban M, Nedelcu R, Turcu G, et al. Epithelial-Mesenchymal Transition in Skin Cancers: A Review. Analytical cellular pathology (Amsterdam). 2019;2019:3851576.

33. Guan S, Zhou J. CXCR7 attenuates the TGF- $\beta$-induced endothelial-to-mesenchymal transition and pulmonary fibrosis. Molecular bioSystems. 2017;13(10):2116-24.

34. Gaines T, Camp D, Bai R, Liang Z, Yoon Y, Shim H, et al. Synthesis and evaluation of 2,5 and 2,6 pyridine-based CXCR4 inhibitors. Bioorg Med Chem. 2016;24(21):5052-60.

35. Harms M, Gilg A, Ständker L, Beer A, Mayer B, Rasche V, et al. Microtiter plate-based antibodycompetition assay to determine binding affinities and plasma/blood stability of CXCR4 ligands. Scientific reports. 2020;10(1):16036.

36. Wang Q, Wang S, Zhang Y, Yu Y, Zhao H, Yang H, et al. The CXC chemokines and CXC chemokine receptors in orange-spotted grouper (Epinephelus coioides) and their expression after Singapore grouper iridovirus infection. Dev Comp Immunol. 2019;90:10-20.

37. Zou Y, Kottmann A, Kuroda M, Taniuchi I, Littman D. Function of the chemokine receptor CXCR4 in haematopoiesis and in cerebellar development. Nature. 1998;393(6685):595-9. 
38. Dinkel B, Kremer K, Rollins M, Medlyn M, Hedin K. GRK2 mediates TCR-induced transactivation of CXCR4 and TCR-CXCR4 complex formation that drives PI3KY/PREX1 signaling and T cell cytokine secretion. J Biol Chem. 2018;293(36):14022-39.

39. Zang J, Ye J, Zhang C, Sha M, Gao J. Senescent hepatocytes enhance natural killer cell activity via the CXCL-10/CXCR3 axis. Experimental therapeutic medicine. 2019;18(5):3845-52.

40. Susek K, Karvouni M, Alici E, Lundqvist A. The Role of CXC Chemokine Receptors 1-4 on Immune Cells in the Tumor Microenvironment. Frontiers in immunology. 2018;9:2159.

41. Zlotnik A, Burkhardt A, Homey B. Homeostatic chemokine receptors and organ-specific metastasis. Nature reviews Immunology. 2011;11(9):597-606.

42. Bektur Aykanat N, Kacar S, Karakaya S, Sahinturk V. Silymarin suppresses HepG2 hepatocarcinoma cell progression through downregulation of Slit-2/Robo-1 pathway. Pharmacol Rep. 2020;72(1):199207.

43. Hartmann N, Giese N, Giese T, Poschke I, Offringa R, Werner J, et al. Prevailing role of contact guidance in intrastromal T-cell trapping in human pancreatic cancer. Clinical cancer research: an official journal of the American Association for Cancer Research. 2014;20(13):3422-33.

44. Chen F, Yuan J, Yan H, Liu H, Yin S. Chemokine Receptor CXCR3 Correlates with Decreased M2 Macrophage Infiltration and Favorable Prognosis in Gastric Cancer. BioMed research international. 2019;2019:6832867.

45. Saxena R, Wang Y, Mier J. CXCR4 inhibition modulates the tumor microenvironment and retards the growth of B16-OVA melanoma and Renca tumors. Melanoma research. 2020;30(1):14-25.

46. Maravillas-Montero J, Burkhardt A, Hevezi P, Carnevale C, Smit M, Zlotnik A. Cutting edge: GPR35/CXCR8 is the receptor of the mucosal chemokine CXCL17. Journal of immunology (Baltimore, Md: 1950). 2015;194(1):29-33.

\section{Tables}

Table $₫$ The similar neighbor genes of CXCRs isolated with GEPIA. 


\begin{tabular}{|ll|}
\hline CXCRs & Similar neighbor genes \\
\hline CXCR1 & $\begin{array}{l}\text { RP11-531H8.2, LINC00694, PRSS3, CSF3, SPANXN4, RP11-217E22.5, CTB-78F1.1, } \\
\text { ACEA_U3, CTD-2015G9.1, RP11-361M10.4 }\end{array}$ \\
\hline CXCR2 & ABCA12, WH43, CYP4F3, GRHL1, PNPLA1, SDR16C5, SPINK5, TMEM45A, UNC93A, ZNF750 \\
\hline CXCR3 & TRAC, SLA2, SIRPG, IL2RB, HCST, CTSW, CD7, CD27, CD2, CCL5 \\
\hline CXCR4 & CYTIP, CD69, TNFSF8, CD48, TAGAP, FAM159A, GPR183, SELL, CD52, CD37 \\
\hline CXCR5 & TREML2, TNFRSF13C, SPIB, PAX5, LTB, CNR2, CLEC17A, CD19, CCR6, BLK \\
\hline CXCR6 & GBP5, CCL4, CD8A, FASLG, TIGIT, CD8B, GZMA, IL2RB, SIRPG, JAKMIP1 \\
\hline CXCR7 & BCL6B, CD93, CDH5, CLEC14A, COL4A1, CXorf36, ESAM, KCNJ8, NID2, PCDH12 \\
\hline
\end{tabular}

\section{Figures}


A

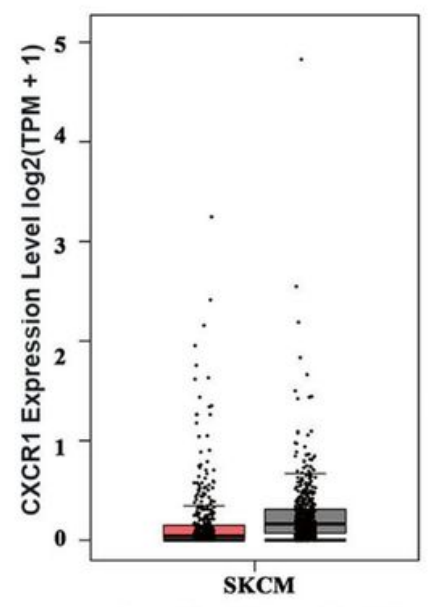

(num $(T)=461 ; \operatorname{num}(N)=558)$

CXCR1

E

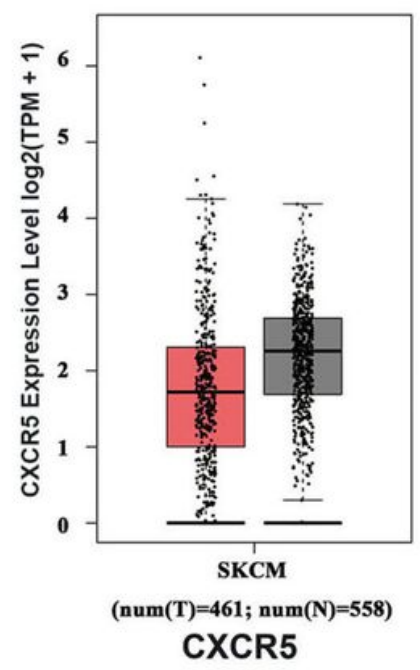

B
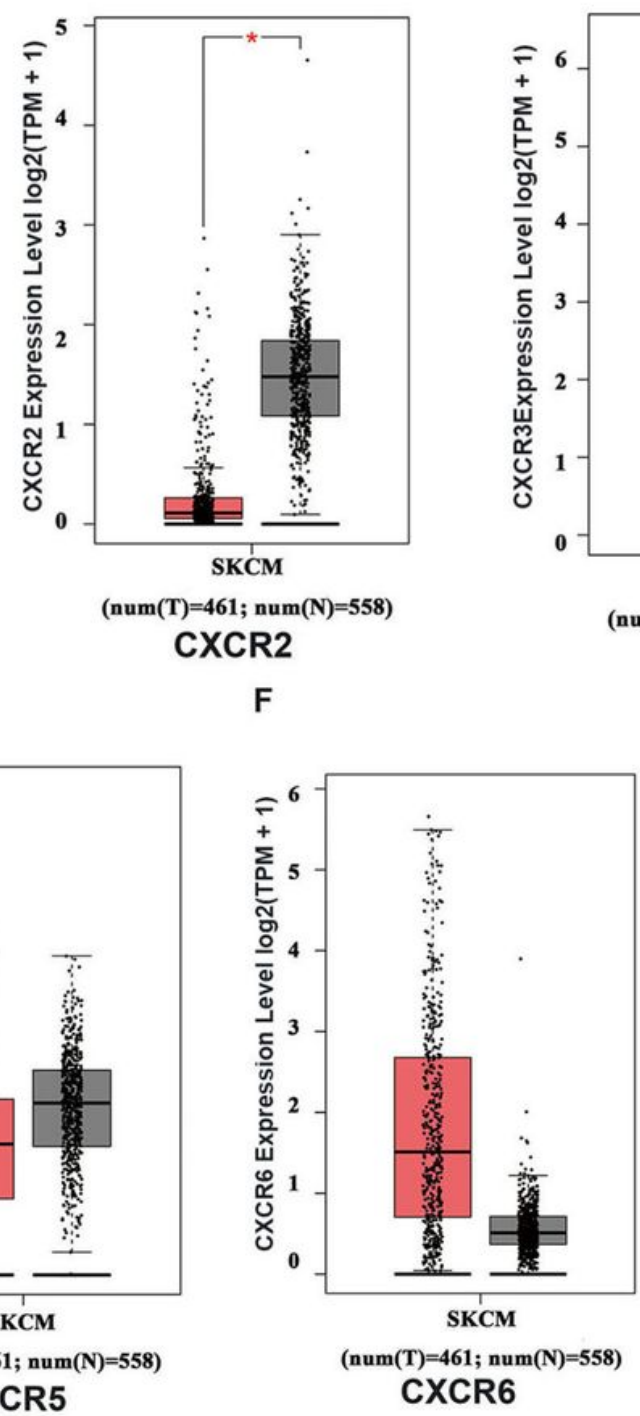

D

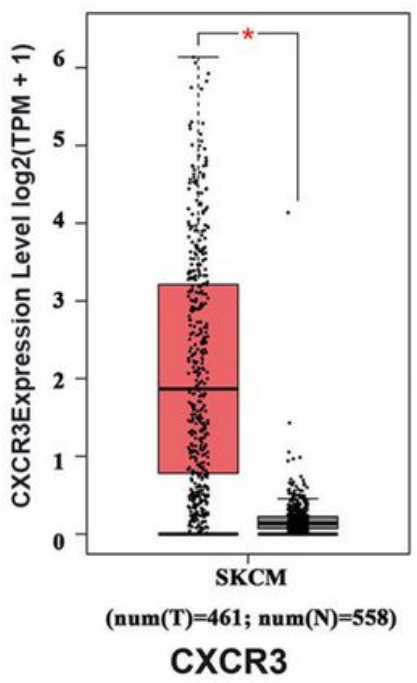

G

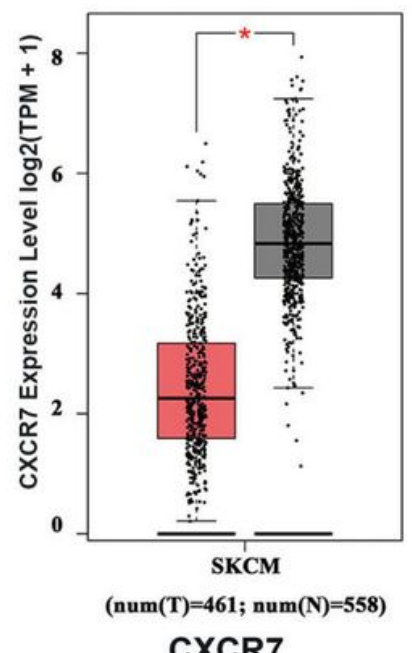

\section{Figure 1}

The expression level of CXCRs in SKCM (GEPIA). T (SKCM tumor tissue); $\mathrm{N}$ (normal tissue). ${ }^{\text {means }}$ the p-value $<0.05$. The transcriptional levels of CXCR3 and CXCR4 in SKCM tissues were significantly elevated, while the transcriptional levels of CXCR2, CXCR7 were significantly reduced. 
A
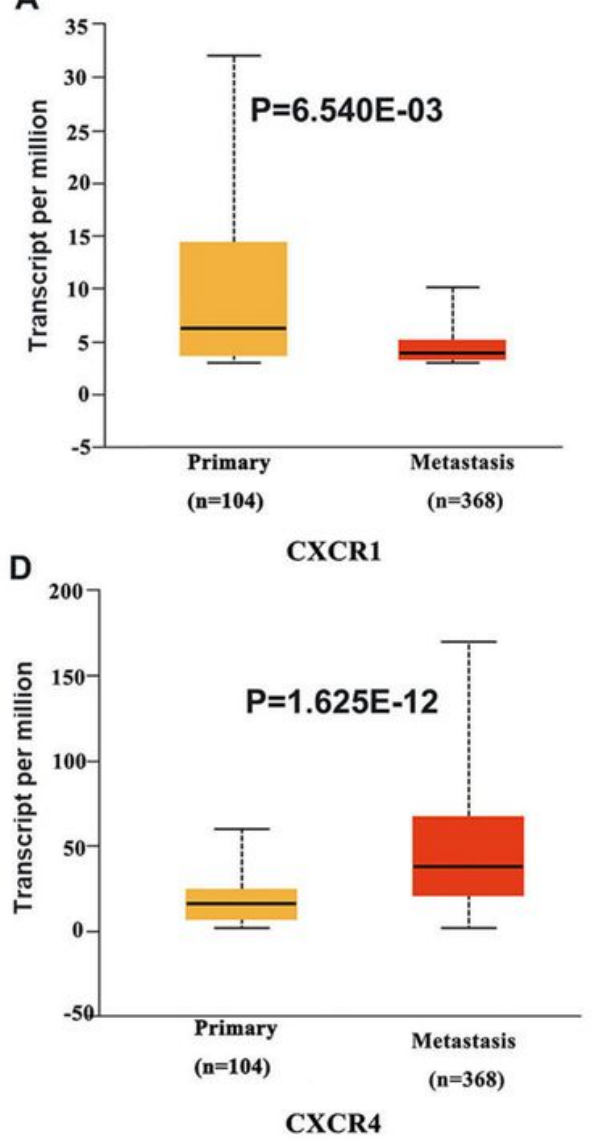

B
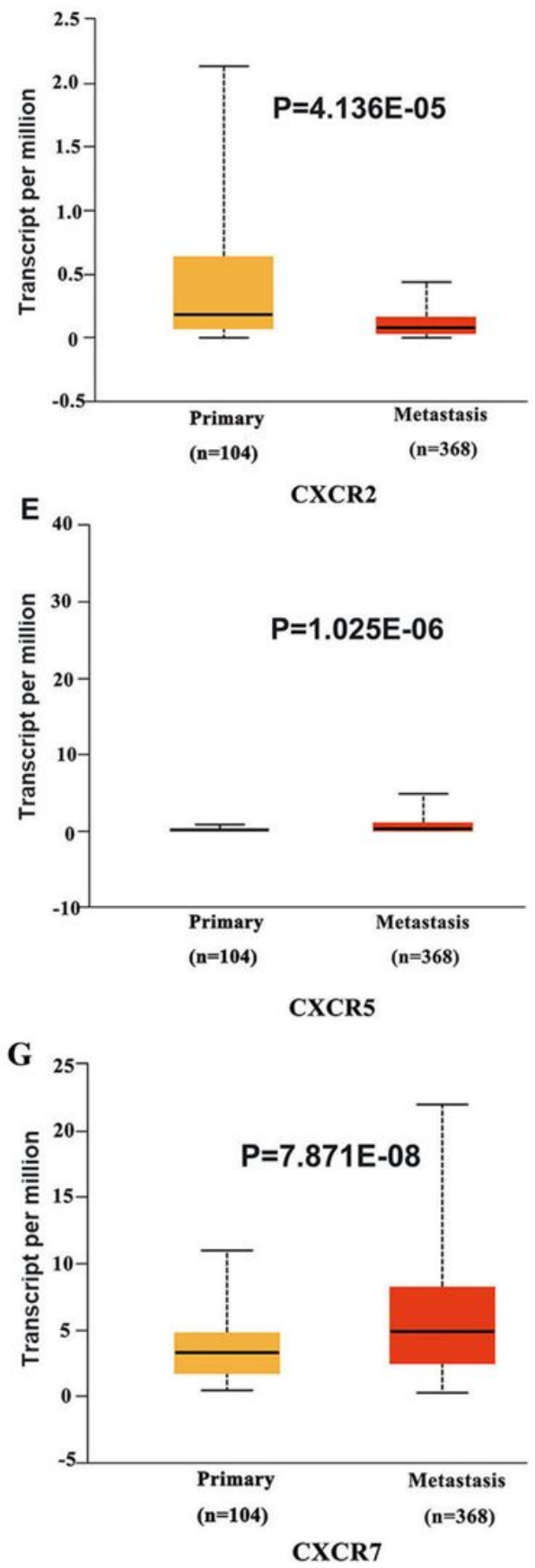

C
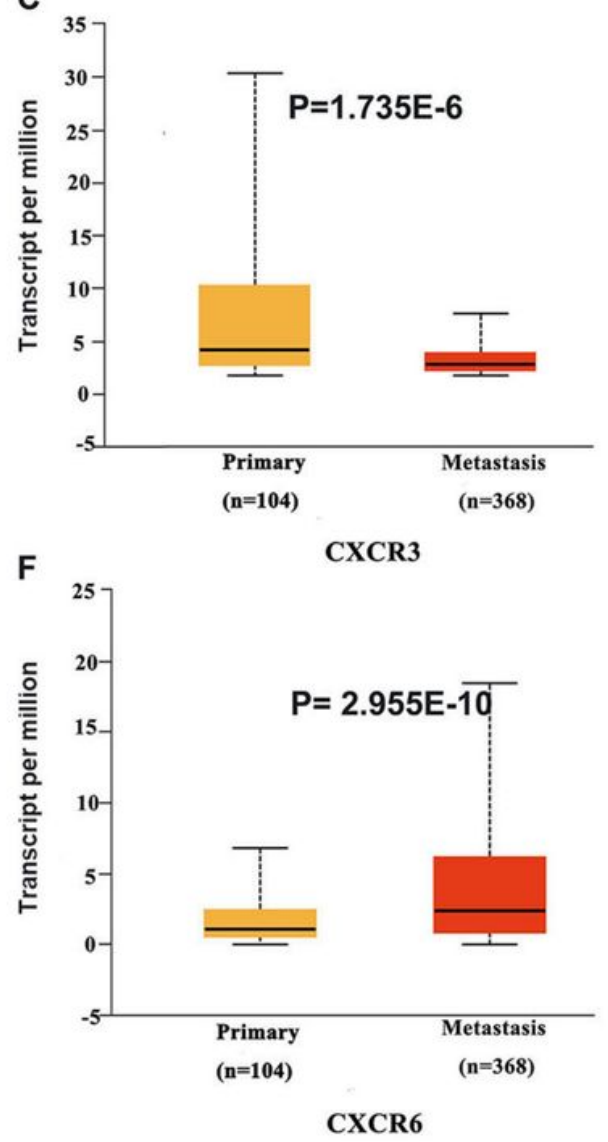

\section{Figure 2}

The mRNA expression of distinct CXCR family members in metastasis SKCM tissues and primary SKCM (GEPIA). The difference in mRNA expression was compared by students' t-test considering unequal variance. The $\mathrm{p}$-value was set at 0.05 . 


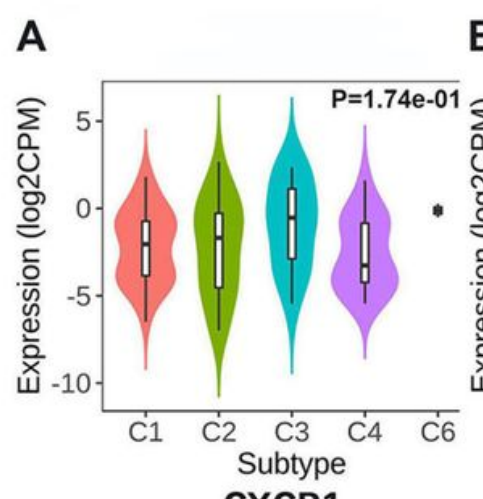

CXCR1
B

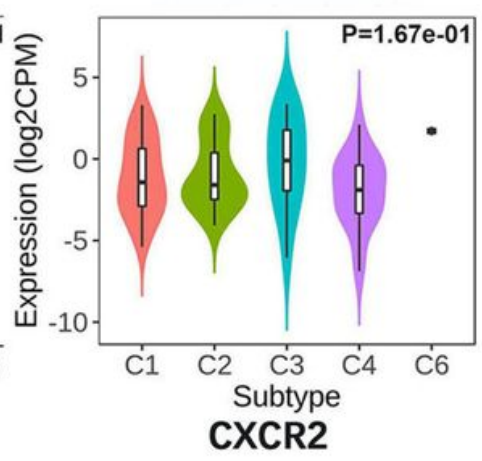

$\mathbf{F}$
C

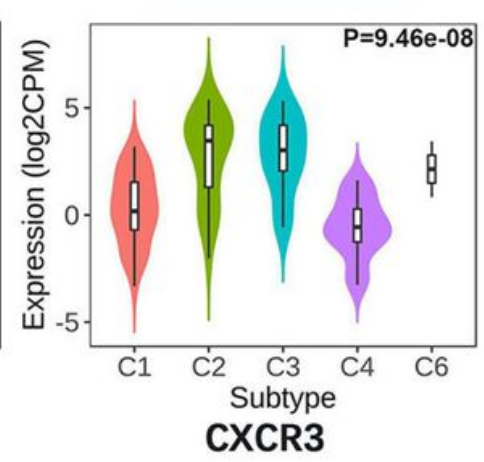

G
D

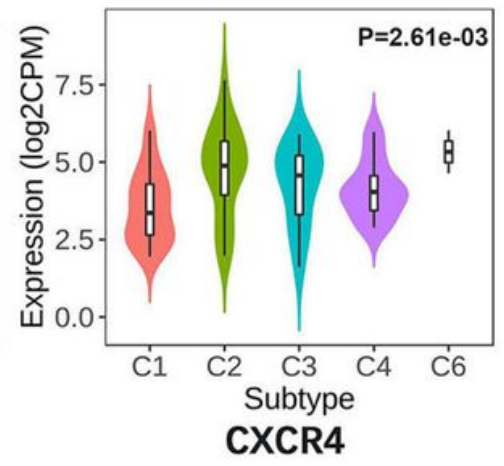

E

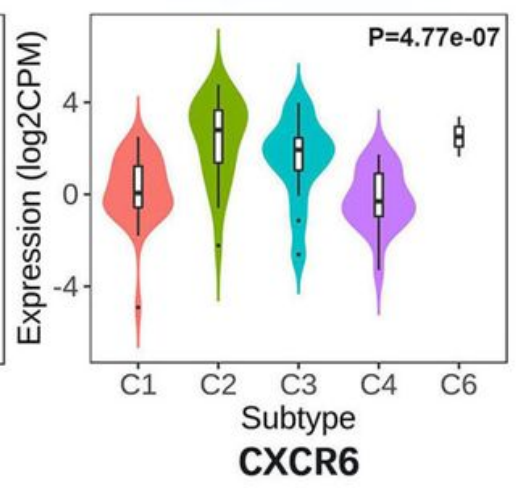

\section{Figure 3}

The distribution of CXCRs across immune subtypes (TISIDB). Statistical significance of differential expression evaluated using Kruskal-Wallis test. C1 (wound healing); C2 (IFN-gamma dominant) C3 (inflammatory); C4 (lymphocyte depleted); C6 (TGF-b dominant). 

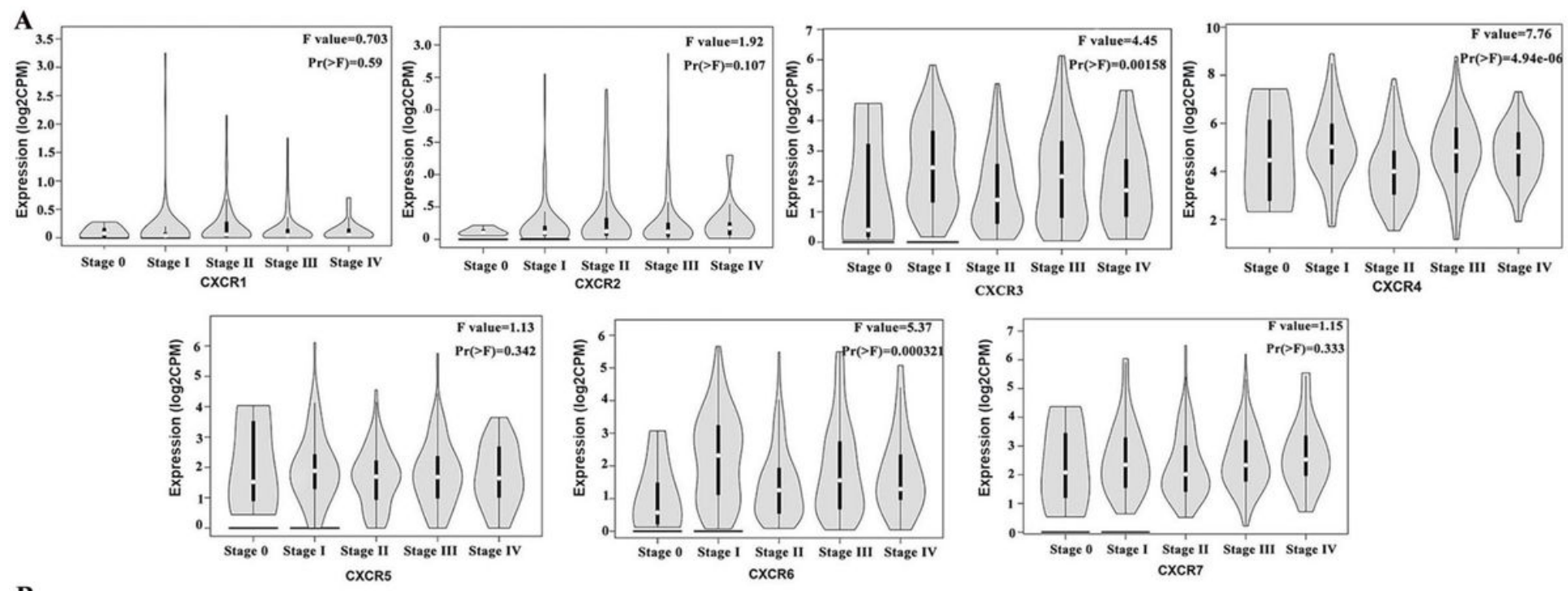

B
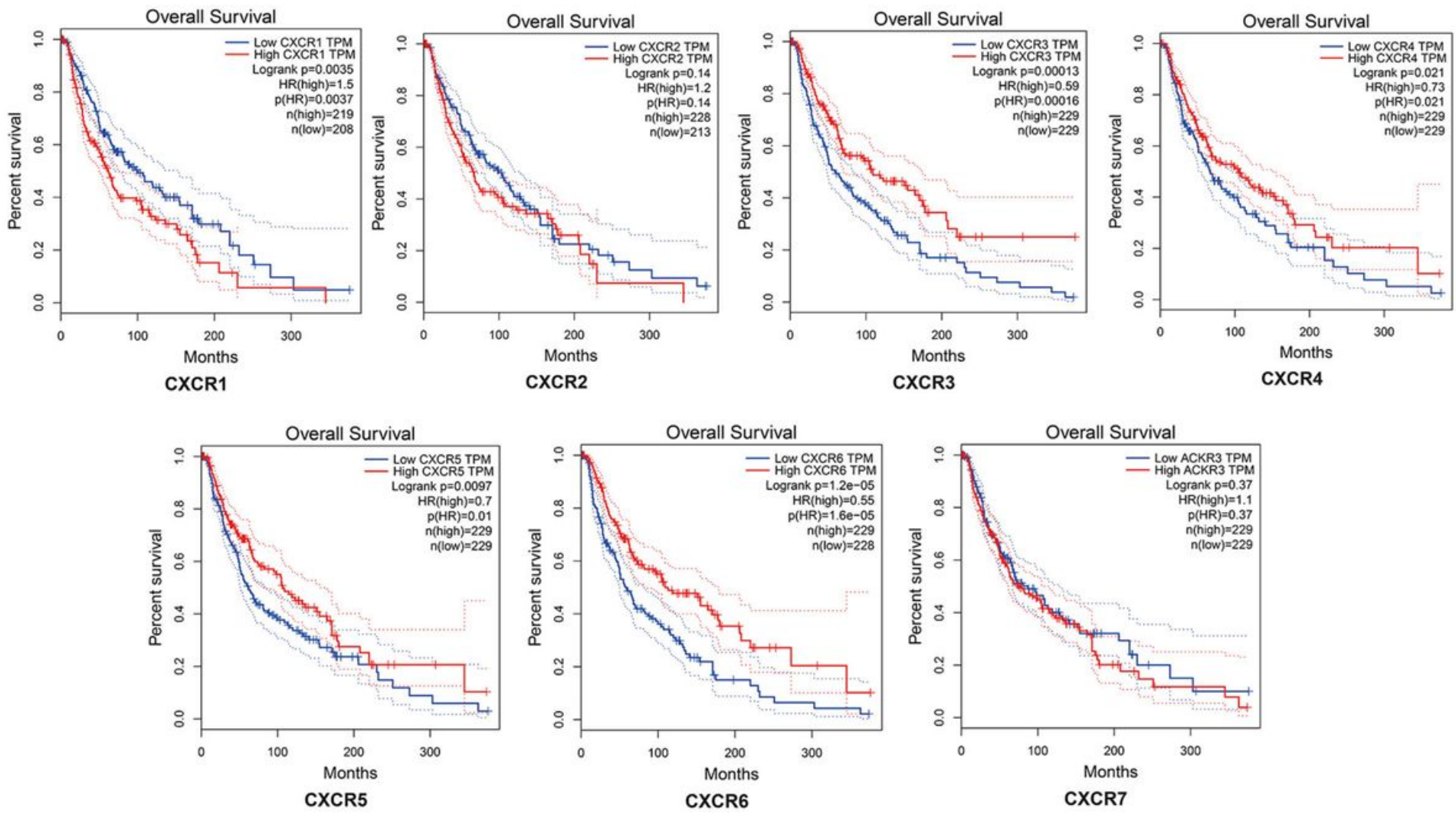

Figure 4

The correlation between CXCRs and the pathological stage, overall survival of SKCM (GEPIA). (A) The expression of CXCR3, CXCR4, and CXCR6 were significantly associated with pathological stage. (B) The disease-free survival curve of CXCRs in SKCM patients. SKCM patients with high expression of CXCR3, CXCR4, CXCR5, and CXCR6, and low expression of CXCR1 had a better overall survival. 

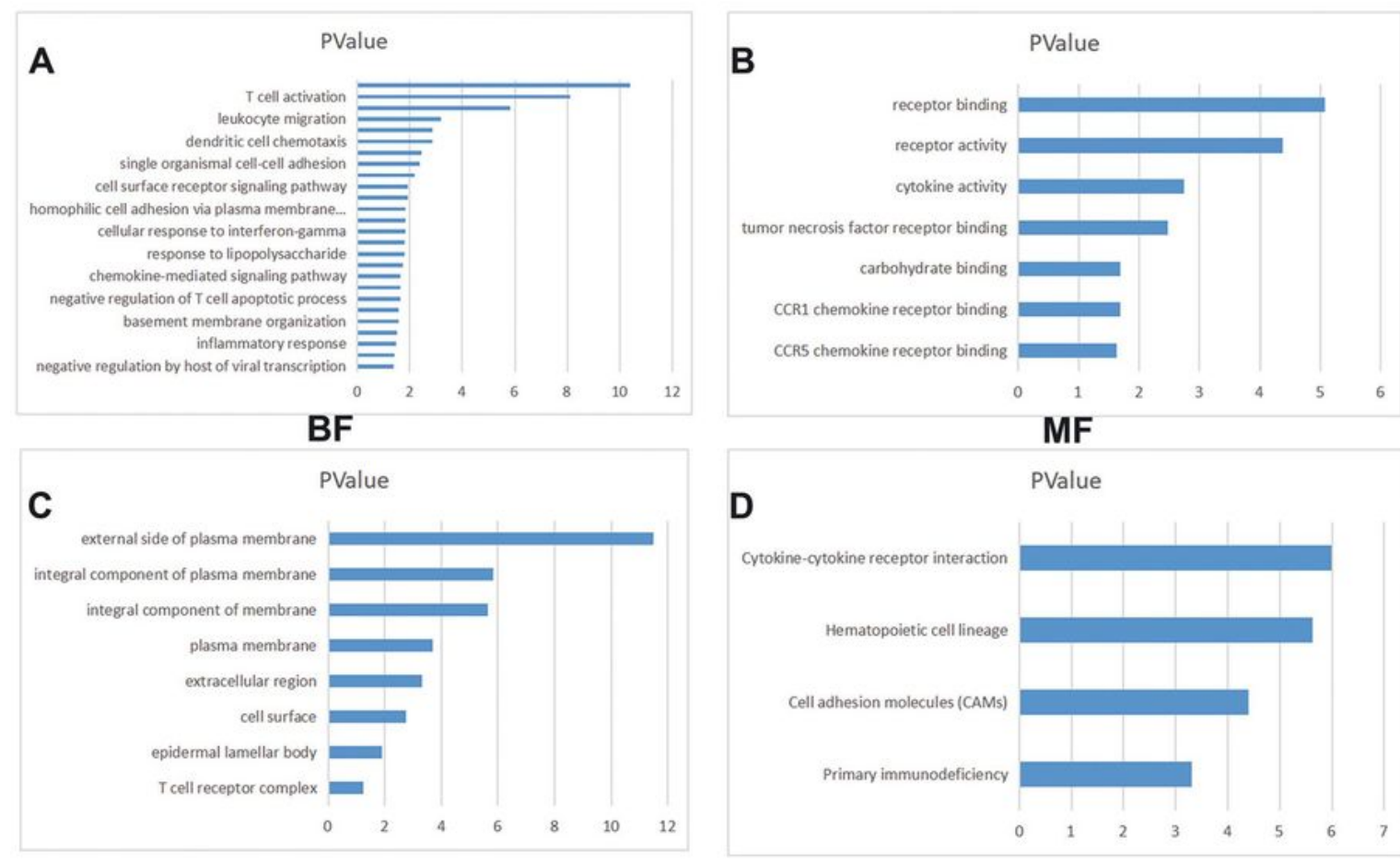

E

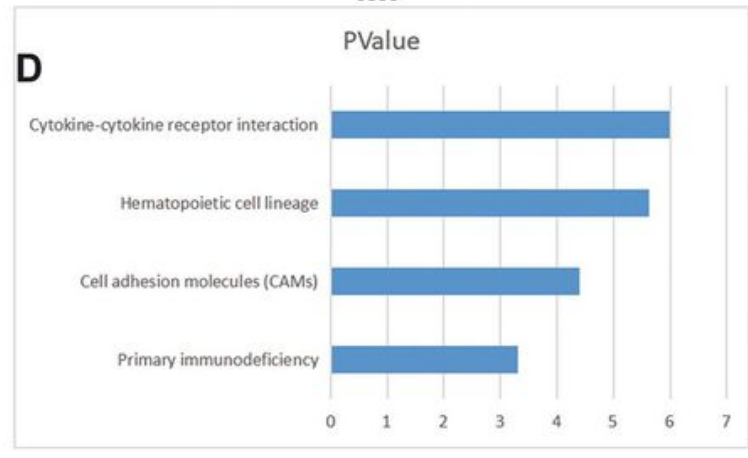

KEGG
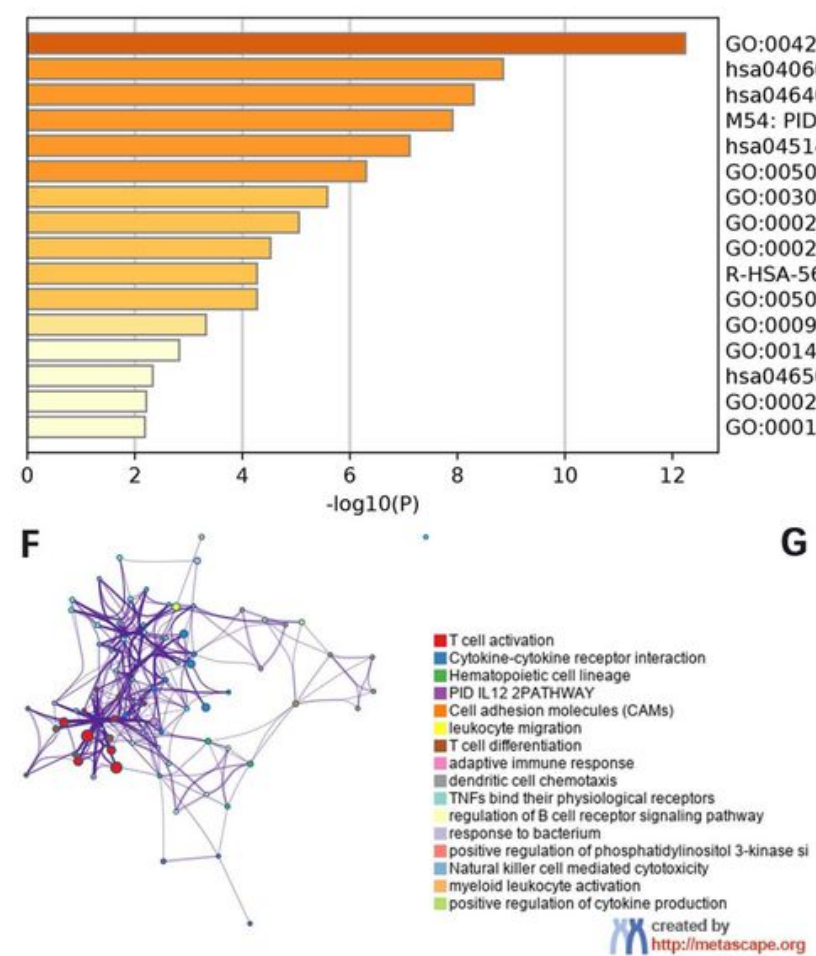

GO:0042110: T cell activation

hsa04060: Cytokine-cytokine receptor interaction

hsa04640: Hematopoietic cell lineage

M54: PID IL12 2PATHWAY

hsa04514: Cell adhesion molecules (CAMs)

GO:0050900: leukocyte migration

GO:0030217: T cell differentiation

GO:0002250: adaptive immune response

GO:0002407: dendritic cell chemotaxis

R-HSA-5669034: TNFs bind their physiological receptors

GO:0050855: regulation of B cell receptor signaling pathway

GO:0009617: response to bacterium

GO:0014068: positive regulation of phosphatidylinositol 3-kinase signaling

hsa 04650: Natural killer cell mediated cytotoxicity

GO:0002274: myeloid leukocyte activation

GO:0001819: positive regulation of cytokine production

G

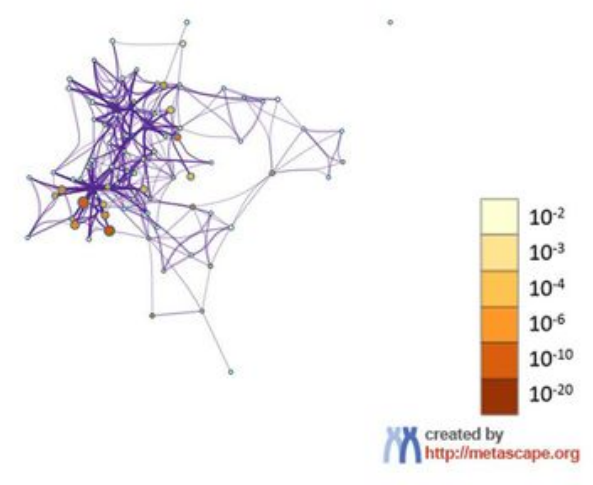

Figure 5

The enrichment analysis of differentially expressed CXCRs and 71 most similar neighbor genes in SKCM using David 6.8 (A-D) and Metascape (E-G). (A) biological process (BP); (B) molecular function (MF); (C) cellular component (CC); (D) KEGG pathway analysis. (E)bar-plot of top 16 enriched terms across CXCRs and neighbor genes, colored by p-values. (F-G) Network of enriched terms, colored by p-values. 
A

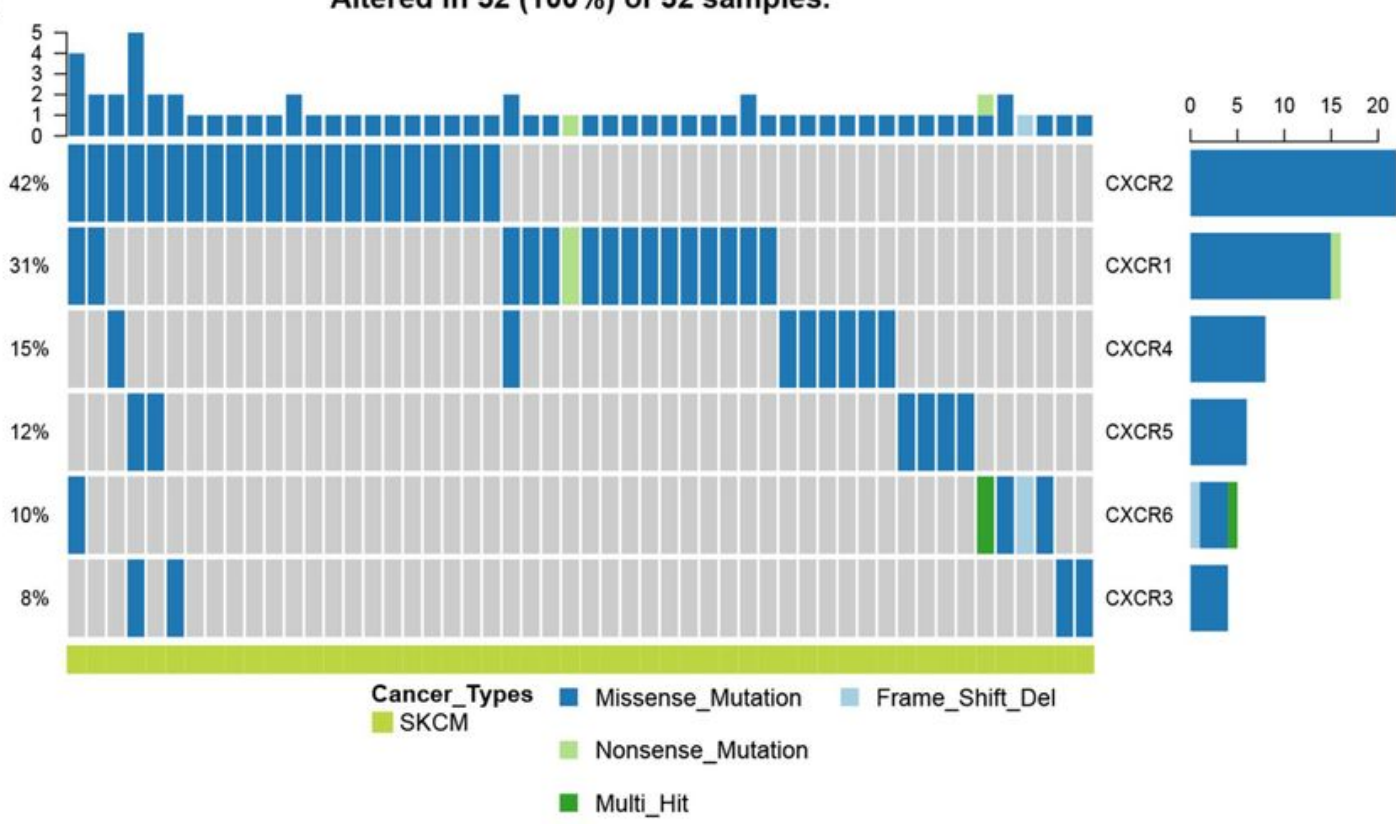

B

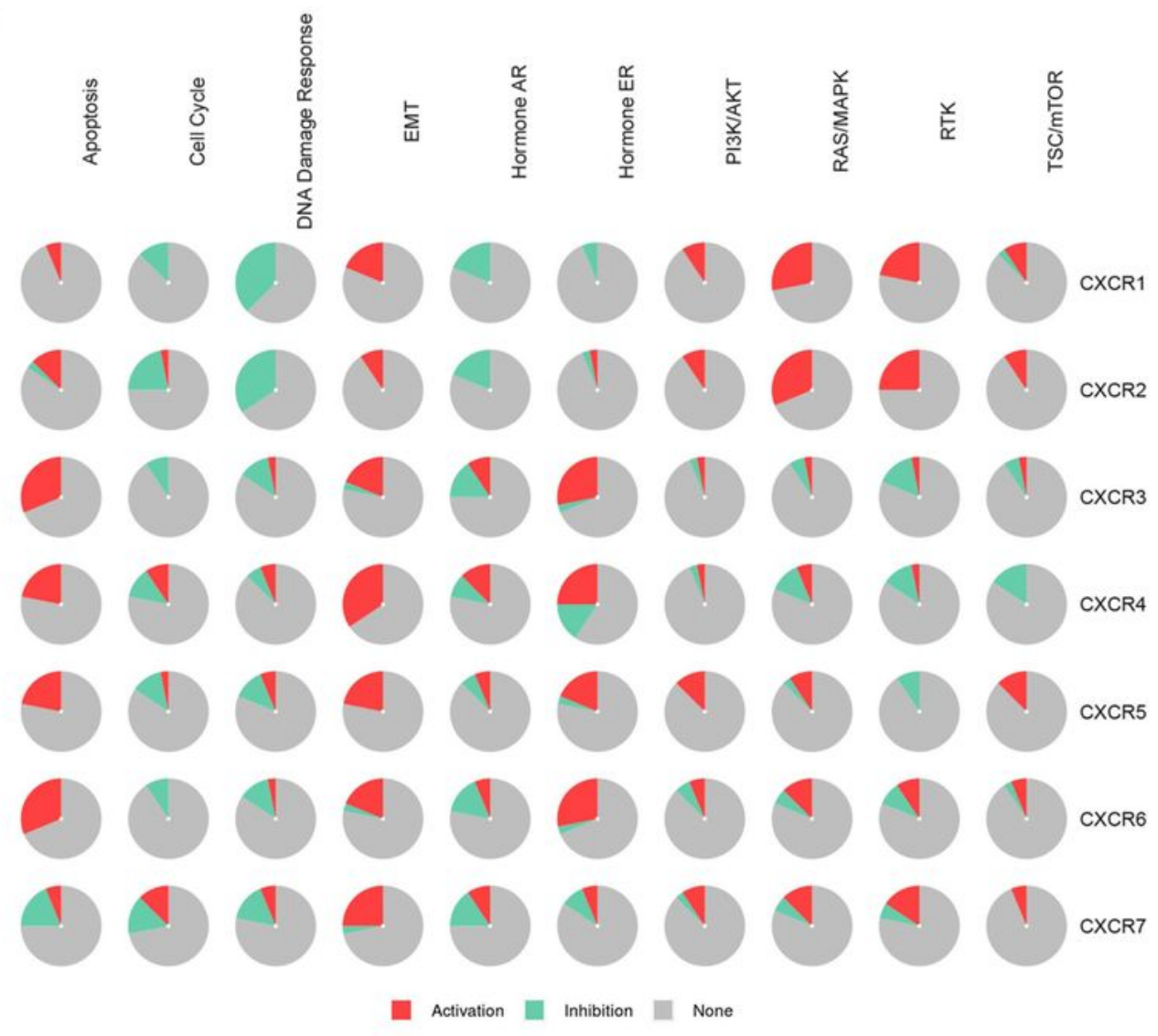

Figure 6

The genetic variation analysis (A) and cancer pathway activity (B) of CXCRs in SKCM patients (GSCALite).(A) Summary plot displays genetic variation frequency and variant types of CXCRs in SKCM. (B) The relationship between pathways and CXCRs. 


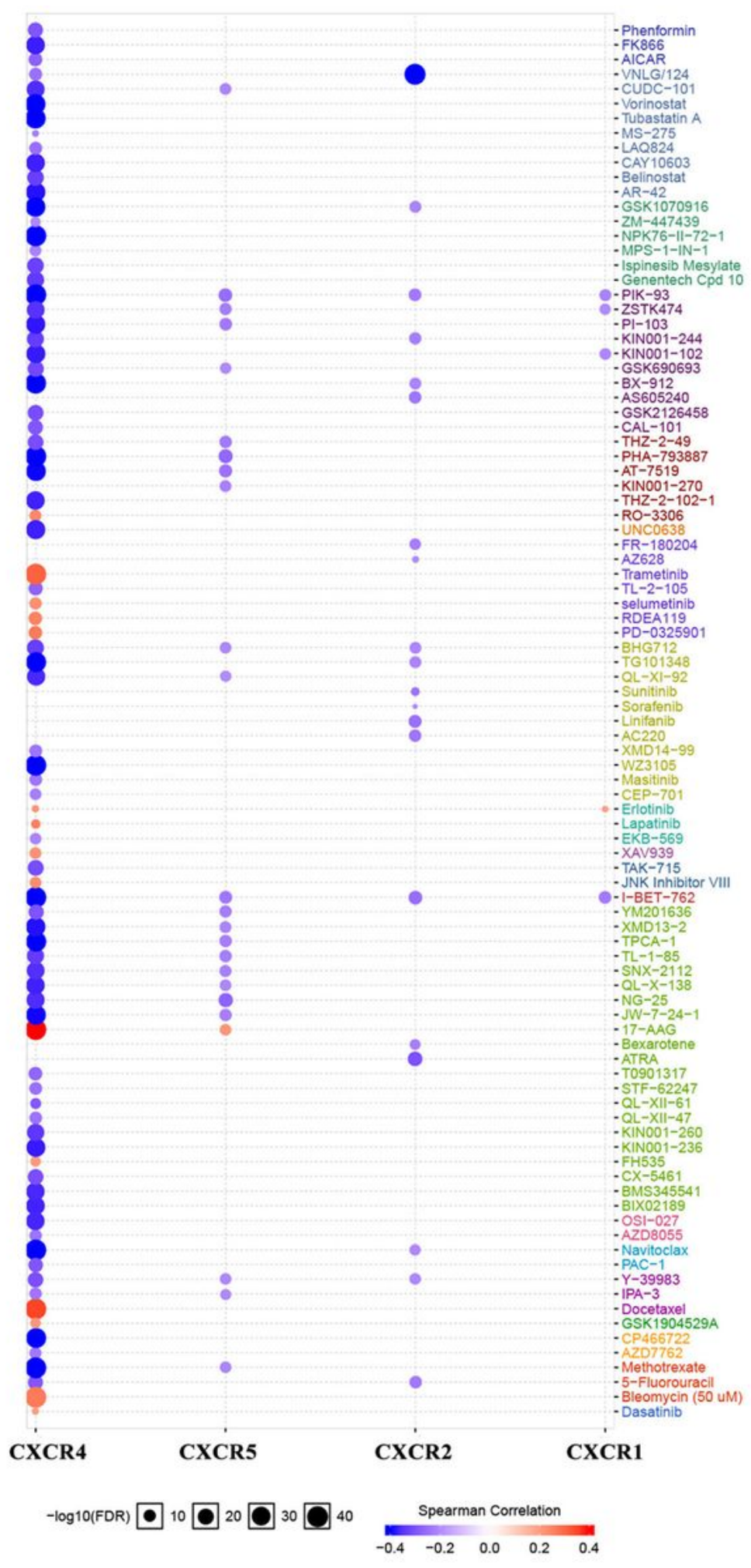

\section{Figure 7}

The drug resistance analysis of CXCRs based on GDSC IC50 drug data. The Spearman correlation represent the gene expression correlates with the drug. The positive correlation means that the gene high expression is resistant to the drug, vise verse. 

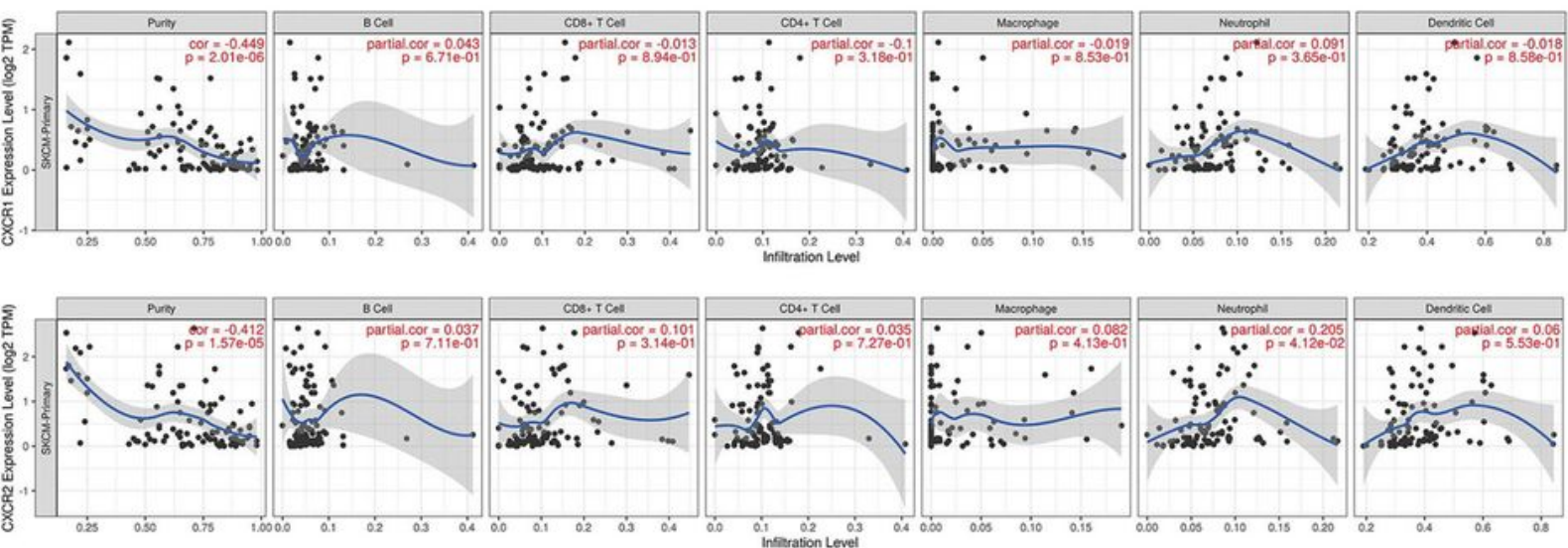

C
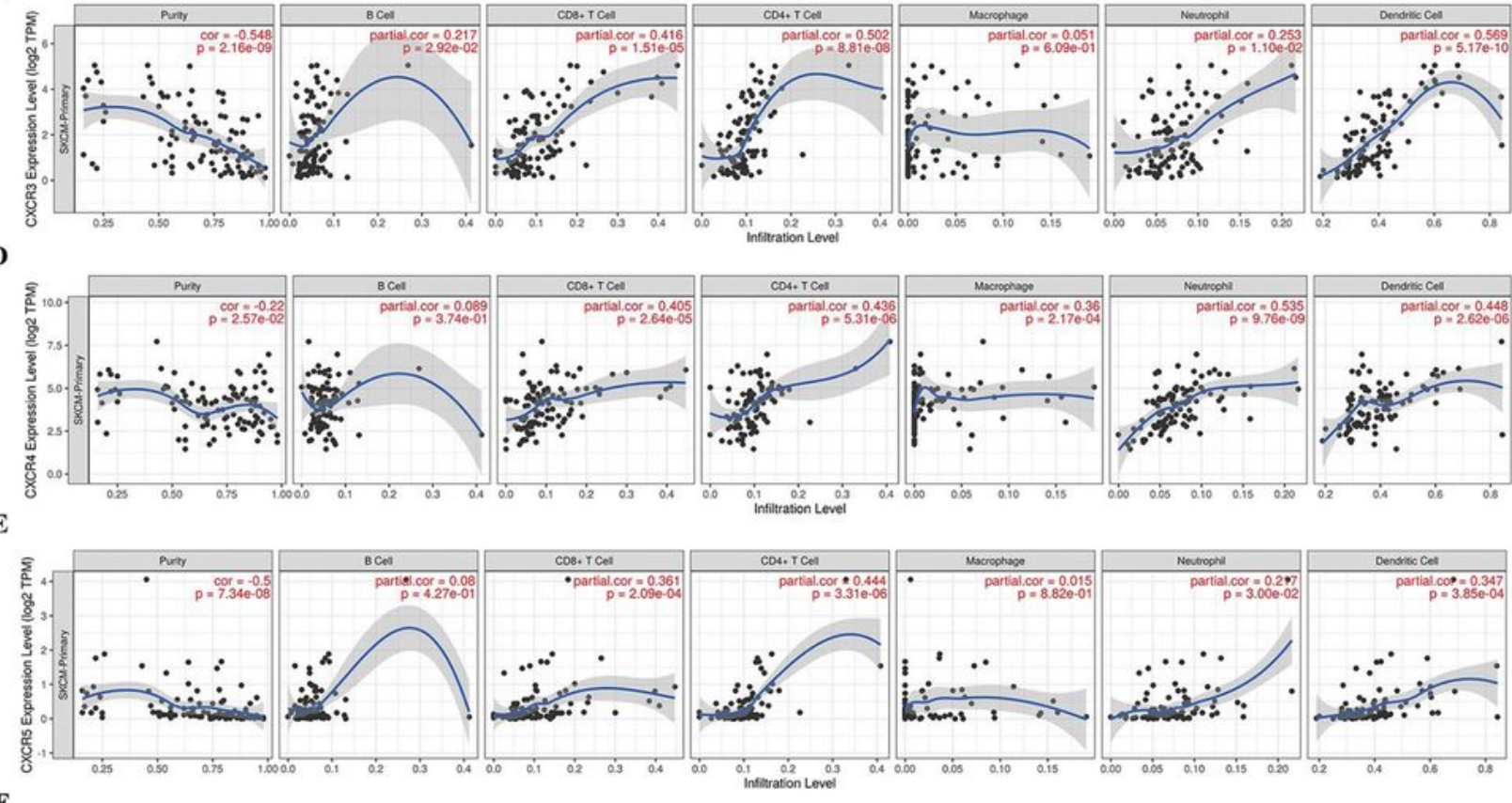

$\mathbf{F}$
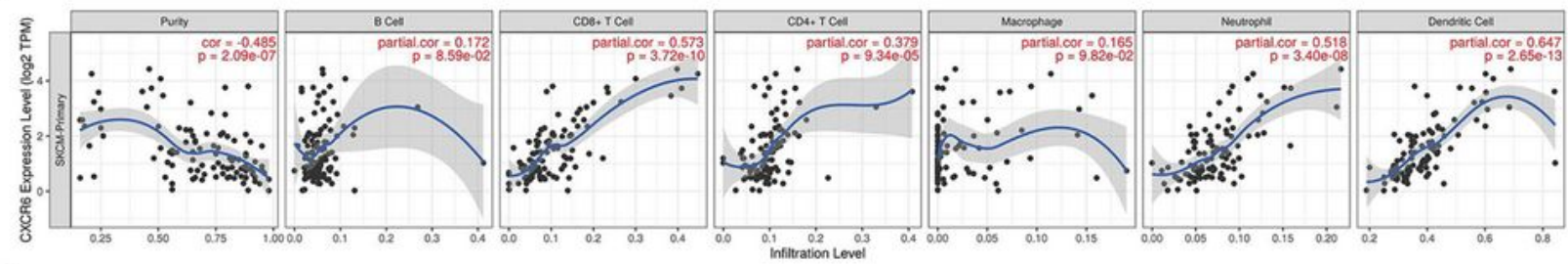

G

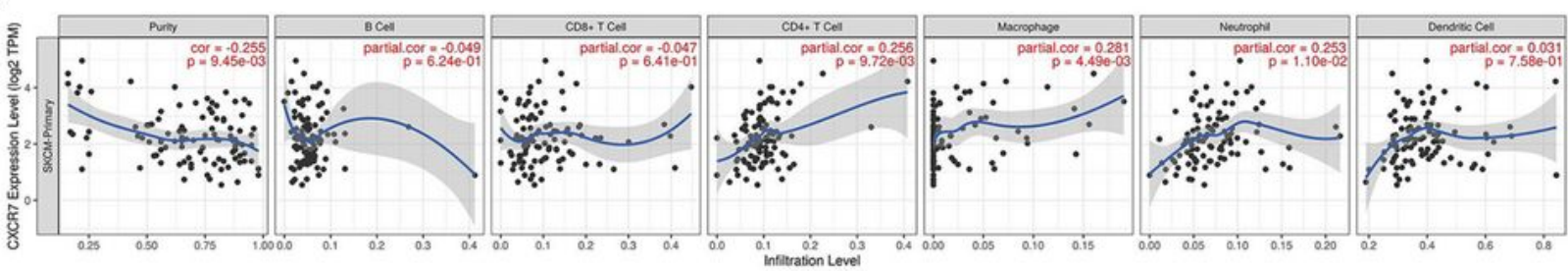

Figure 8

The correlation between different expressed CXCRs and immune cell infiltration. The correlation was adjusted by purity. 

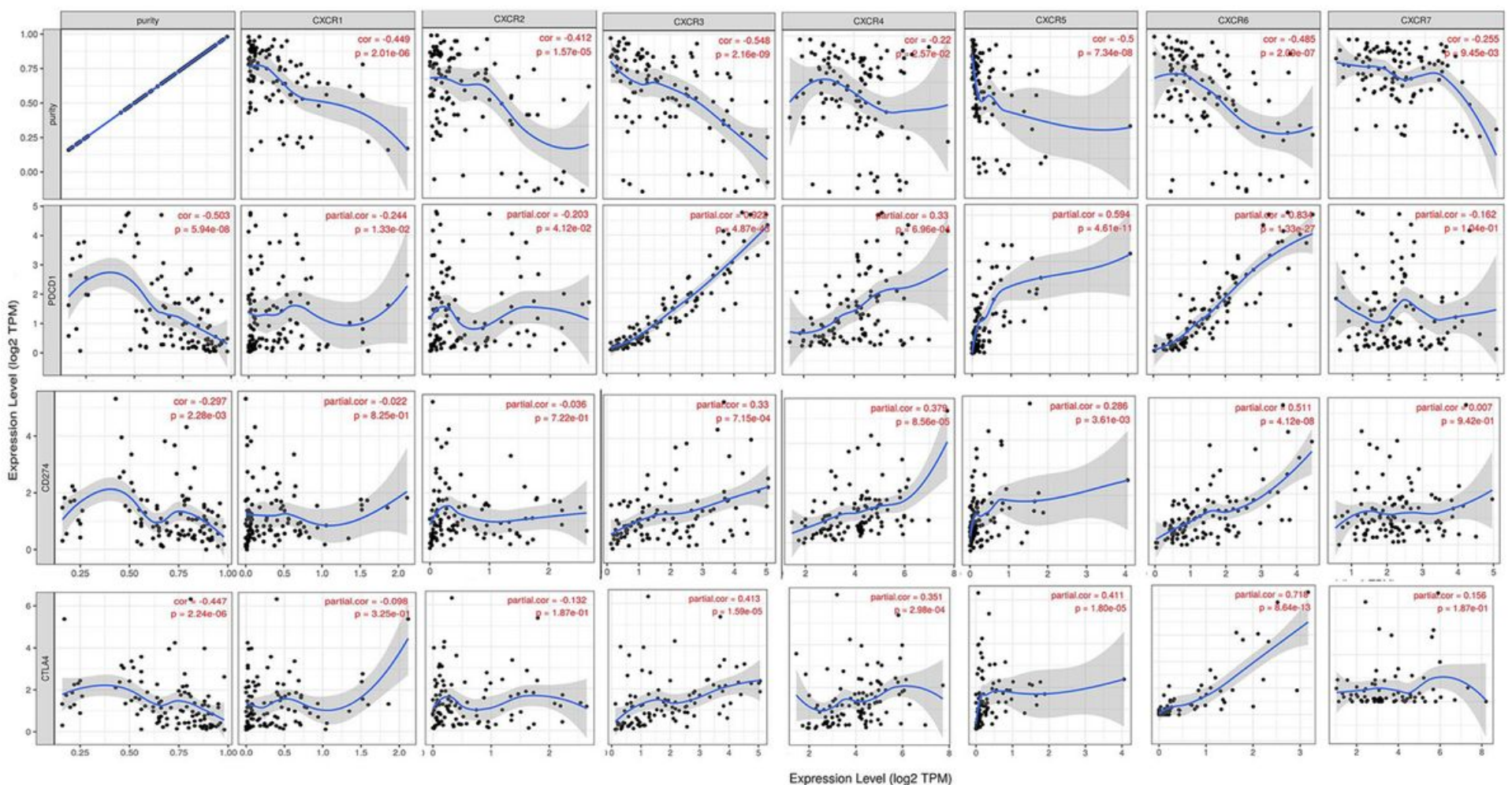

Figure 9

The correlation between different expressed CXCRs and PD-1, PD-L1, and CTLA-4 in SKCM (TIMER). The correlation was adjusted by purity. 